\title{
The A-integral and Restricted Riesz Transform
}

\author{
Rashid A. AliEV* AND Khanim I. NebiYeVA
}

ABSTRACT. It is known that the restricted Riesz transform of a Lebesgue integrable function is not Lebesgue integrable. In this paper, we prove that the restricted Riesz transform of a Lebesgue integrable function is $A$-integrable and the analogue of Riesz's equality holds.

Keywords: Riesz transform, $A$-integral, Riesz's equality, covering theorem, singular integrals.

2010 Mathematics Subject Classification: 44A15, 42B20, 26A39, 26 B10.

\section{INTRODUCTION}

The $j$-th Riesz transform of a function $f \in L_{p}\left(R^{d}\right), 1 \leq p<+\infty$ is defined as the following singular integral:

$$
R_{j}(f)(x)=C_{(d)} \lim _{\varepsilon \rightarrow 0} \int_{\left\{y \in R^{d}:|x-y|>\varepsilon\right\}} \frac{x_{j}-y_{j}}{|x-y|^{d+1}} f(y) d y, j=\overline{1, d},
$$

where $C_{(d)}=\frac{\Gamma((d+1) / 2)}{\pi^{(d+1) / 2}}, \Gamma(z)=\int_{0}^{+\infty} t^{z-1} e^{-t} d t$ - Euler Gamma function and $d$ - dimension of the space $R^{d}$.

Let $\Omega \subset R^{d}$ be a bounded domain and $f \in L_{1}(\Omega)$. In the present paper, we consider the corresponding modification of $R_{j}$. Namely, the restricted Riesz transform $R_{j, \Omega}$ is defined as

$$
\begin{gathered}
\left(R_{j, \Omega} f\right)(z)=R_{j}\left(\chi_{\Omega} f\right)(z) \\
=C_{(d)} \lim _{\varepsilon \rightarrow 0} \int_{\{y \in \Omega:|x-y|>\varepsilon\}} \frac{x_{j}-y_{j}}{|x-y|^{d+1}} f(y) d y, j=\overline{1, d}, z \in \Omega,
\end{gathered}
$$

where $\chi_{\Omega}(z)=1$ for $z \in \Omega$ and $\chi_{\Omega}(z)=0$ for $z \in R^{d} \backslash \Omega$ is the characteristic function of the set $\Omega$.

From the theory of singular integrals (see [15]) it is known that the Riesz transform is a bounded operator in the space $L_{p}(\Omega), p>1$, that is, if $f \in L_{p}(\Omega)$, then $R_{j, \Omega}(f) \in L_{p}(\Omega)$ and the inequality

$$
\left\|R_{j, \Omega} f\right\|_{L_{p}} \leq C^{(p)}\|f\|_{L_{p}}
$$

holds. In the case $f \in L_{1}(\Omega)$ only the weak inequality holds:

$$
m\left\{x \in \Omega:\left|\left(R_{j, \Omega} f\right)(x)\right|>\lambda\right\} \leq \frac{C_{1}}{\lambda}\|f\|_{L_{1}},
$$

Received: 28.04.2020; Accepted: 10.08.2020; Published Online: 16.08.2020

*Corresponding author: Rashid A. Aliev; aliyevrashid@mail.ru

DOI: $10.33205 / \mathrm{cma} .728156$ 
where $m$ stands for the Lebesgue measure, $C^{(p)}, C_{1}$ are constants independent of $f$. From the inequalities (1.1), (1.2) it follows that the Riesz transform of the function $f \in L_{1}(\Omega)$ satisfies the condition

$$
m\left\{x \in \Omega:\left|\left(R_{j, \Omega} f\right)(x)\right|>\lambda\right\}=o\left(\frac{1}{\lambda}\right), \lambda \rightarrow+\infty .
$$

Note that the Riesz transform of a function $f \in L_{1}(\Omega)$ is not Lebesgue integrable. In this paper, we prove that the Riesz transform of a function $f \in L_{1}(\Omega)$ is $A$-integrable on $\Omega$ and the analogue of Riesz's equality holds.

\section{2. $A$ - INTEGRAL}

For a measurable complex function $f(x)$ on domain $\Omega$ we set

$$
\begin{gathered}
{[f(x)]_{n}=[f(x)]^{n}=f(x) \text { for }|f(x)| \leq n} \\
{[f(x)]_{n}=n \operatorname{sgn} f(x),[f(x)]^{n}=0 \text { for }|f(x)|>n, n \in N,}
\end{gathered}
$$

where $\operatorname{sgn} z=z /|z|$ for $z \neq 0$ and $\operatorname{sgn} 0=0$.

In 1929, E.Titchmarsh [16] introduced the notions of $Q$ - and $Q^{\prime}$-integrals of function, measurable on $\Omega$.

Definition 2.1. If a finite limit $\lim _{n \rightarrow \infty} \int_{\Omega}[f(x)]_{n} d x\left(\lim _{n \rightarrow \infty} \int_{\Omega}[f(x)]^{n} d x\right.$, respectively) exists, then $f$ is said to be $Q$-integrable ( $Q^{\prime}$-integrable, respectively) on $\Omega$, that is $f \in Q(\Omega)\left(f \in Q^{\prime}(\Omega)\right)$, and the value of this limit is referred to as the $Q$-integral $\left(Q^{\prime}\right.$-integral) of this function and is denoted by

$$
\text { (Q) } \int_{\Omega} f(x) d x\left(\left(Q^{\prime}\right) \int_{\Omega} f(x) d x\right) \text {. }
$$

In the same paper, E.Titchmarsh established that, when studying the properties of trigonometric series conjugate to Fourier series of Lebesgue integrable functions, $Q$-integration leads to a series of natural results. A very uncomfortable fact impeding the application of $Q$-integrals and $Q^{\prime}$-integrals when studying diverse problems of function theory is the absence of the additivity property, that is, the $Q$-integrability ( $Q^{\prime}$-integrability) of two functions does not imply the $Q$-integrability $\left(Q^{\prime}\right.$-integrability) of their sum. If one adds the condition

$$
m\{z \in \Omega:|f(x)|>\lambda\}=o\left(\frac{1}{\lambda}\right), \lambda \rightarrow+\infty
$$

to the definition of $Q$-integrability ( $Q^{\prime}$-integrability) of a function $f$, then the $Q$-integral and $Q^{\prime}$-integral coincide $\left(Q(\Omega)=Q^{\prime}(\Omega)\right)$, and these integrals become additive.

Definition 2.2. If $f \in Q^{\prime}(\Omega)$ (or $f \in Q(\Omega)$ ) and condition (2.3) holds, then $f$ is said to be $A$-integrable on $\Omega, f \in A(\Omega)$, and the limit $\lim _{n \rightarrow \infty} \int_{\Omega}[f(x)]_{n} d x$ (or the limit $\lim _{n \rightarrow \infty} \int_{\Omega}[f(x)]^{n} d x$ ) is denoted in this case by

$$
\text { (A) } \int_{\Omega} f(x) d x \text {. }
$$

The properties of $Q$ - and $Q^{\prime}$-integrals were investigated in $[2,8,9,16,17]$, and for the applications of $A^{-}, Q$ - and $Q^{\prime}$-integrals in the theory of functions of real and complex variables, covering and flattering arguments, we refer the reader to $[1,2,3,4,5,6,11,12,13,14,17,18]$ and references therein. 


\section{3. $A$ - INTEGRABILITY AND RIESZ'S EQUALITY FOR THE RIESZ TRANSFORM OF LEBESGUE INTEGRABLE FUNCTIONS}

From the properties of singular integrals it follows that (see [15]) if $f \in L_{p}(\Omega), p>1$ and $g \in L_{q}(\Omega), q>1,1 / p+1 / q=1$, then

$$
\begin{gathered}
\int_{\Omega} g(x)\left(R_{j, \Omega} f\right)(x) d x \\
=C_{d} \lim _{\varepsilon \rightarrow 0} \iint_{\{x, y \in \Omega:|x-y|>\varepsilon\}} \frac{x_{j}-y_{j}}{|x-y|^{d+1}} f(y) g(x) d y d x \\
=-\int_{\Omega} f(x)\left(R_{j, \Omega} g\right)(x) d x .
\end{gathered}
$$

In this section, we prove that the Riesz transform of the function $f \in L_{1}(\Omega)$ is $A$-integrable on $\Omega$ and the analogue of (3.1) holds.

Theorem 3.1. Let $f \in L_{1}(\Omega)$ and $g(x)$ is a bounded function on $\Omega$ such that the $\left(R_{j, \Omega} g\right)(x)$ is also bounded on $\Omega$. Then the function $g(x) \cdot\left(R_{j, \Omega} f\right)(x)$ is $A$-integrable on $\Omega$ and the equation

$$
\text { (A) } \int_{\Omega} g(x)\left(R_{j, \Omega} f\right)(x) d x=-\int_{\Omega} f(x)\left(R_{j, \Omega} g\right)(x) d x .
$$

holds.

Proof. Since the $A$-integral satisfies the additivity property, it can be assumed that the function $f$ is real, $f(x) \geq 0$ for any $x \in \Omega$, and

$$
\max _{x \in \Omega}\left\{|g(x)|,\left|\left(R_{j, \Omega} g\right)(x)\right|\right\} \leq 1 .
$$

For $x \notin \Omega$, we assume that $f(x)=0$.

Our proof will depend on a certain refinement of Besicovitch's method [7] for a direct proof of the existence of conjugate function (this method employs only the machinery of the theory of sets of points). This method was improved by Titchmarsh [16] and Ul'yanov [17] for the study of properties of the conjugate function. It is worth noting that Besicovitch-TitchmarshUl'yanov's method is applicable only to functions of one real variable (because this method relies on some facts that are valid only in one-dimensional case). For example, it depends on the fact that any open set is a union of at most a countable number of intervals (to overcome this difficulty, we used Vitali's covering theorem). To make this method to work in the setting of functions of several variables, we have slightly improved the construction.

Denote $\Phi_{n}(x)=f(x)-[f(x)]^{n}$. Then $\alpha_{n}=\int_{\Omega} \Phi_{n}(x) d x \rightarrow 0$ at $n \rightarrow \infty$. Take $n \in N$ such that $\alpha_{n}<1$. Let $E_{n}=\{x \in \Omega: f(x)>n\}$. For any $x \in E_{n}$, we set

$$
r_{x}=\sup \left\{r>0: \int_{B(x ; r)} \Phi_{n}(y) d y=\frac{1}{2} \cdot \frac{\pi^{d / 2}}{\Gamma(1+d / 2)} r^{d} \cdot n\right\}
$$

if $\left\{r>0: \int_{B(x ; r)} \Phi_{n}(y) d y=\frac{1}{2} \cdot \frac{\pi^{d / 2}}{\Gamma(1+d / 2)} r^{d} \cdot n\right\} \neq \emptyset$, and $r_{x}=0$ otherwise, where $B(x ; r)$ - open ball with center at $x$ and with radius $r>0$. Note that if $x \in E_{n}$ is a Lebesgue point of the function $\Phi_{n}(x)$, then $r_{x}>0$ and, therefore, the set $E_{n} \backslash E_{n}^{\prime}$ has a zero measure, where $E_{n}^{\prime}=\left\{x \in E_{n}: r_{x}>0\right\}$.

Consider the system of sets $\left\{B\left(x ; r_{x}\right)\right\}_{x \in E_{n}^{\prime}}$. It follows from the covering theorem (see [10]) that there exists at most a countable points $x_{k} \in E_{n}^{\prime}, k \in I \subset N$ such that the balls $B\left(x_{k} ; r_{x_{k}}\right)$, 
$k \in I$ are pairwise disjoint and

$$
\bigcup_{x \in E_{n}^{\prime}} B\left(x ; r_{x}\right) \subset \bigcup_{k \in I} B\left(x_{k} ; 5 r_{x_{k}}\right) .
$$

Denote

$$
\begin{gathered}
G_{1}=B\left(x_{1} ; 5 r_{x_{1}}\right) \backslash \bigcup_{k>1} B\left(x_{k} ; r_{x_{k}}\right) \\
G_{p}=B\left(x_{p} ; 5 r_{x_{p}}\right) \backslash\left[\bigcup_{j=1}^{p-1} G_{j} \bigcup\left(\bigcup_{k>p} B\left(x_{k} ; r_{x_{k}}\right)\right)\right], p \geq 2, p \in I .
\end{gathered}
$$

Then, the measurable sets $G_{p}, p \in I$ are pairwise disjoint, and

$$
\begin{gathered}
B\left(x_{p} ; r_{x_{p}}\right) \subset G_{p} \subset B\left(x_{p} ; 5 r_{x_{p}}\right), p \in I, \\
E_{n}^{\prime} \subset \bigcup_{x \in E_{n}} B\left(x ; r_{x}\right) \subset \bigcup_{p \in I} G_{p}=\bigcup_{p \in I} B\left(x_{p} ; 5 r_{x_{p}}\right) .
\end{gathered}
$$

Denote $\Phi_{n}^{*}(x)=\frac{1}{m\left(G_{p}\right)} \int_{G_{p}} \Phi_{n}(y) d y$ for $z \in G_{p}, p \in I$ and $\Phi_{n}^{*}(x)=0$ for $x \in R^{d} \backslash \bigcup_{p \in I} G_{p}$. Then for any $p \in I$, we have

$$
\int_{G_{p}} \Phi_{n}(x) d x=\int_{G_{p}} \Phi_{n}^{*}(x) d x .
$$

Note that for any $x \in G_{p}, p \in I$, the inequalities

$$
\begin{aligned}
0 \leq & \Phi_{n}^{*}(x) \leq \frac{1}{m\left(B\left(x_{p} ; r_{x_{p}}\right)\right)} \int_{B\left(x_{p} ; 5 r_{x_{p}}\right)} \Phi_{n}(y) d y \\
& \leq \frac{\Gamma(1+d / 2)}{\pi^{d / 2} r_{x_{p}}^{d}} \cdot \frac{1}{2} \frac{\pi^{d / 2} \cdot 5^{d} r_{x_{p}}^{d}}{\Gamma(1+d / 2)} \cdot n=\frac{5^{d} n}{2}
\end{aligned}
$$

hold. Denote $L_{n}=\bigcup_{p \in I} G_{p}, L_{n}^{\prime}=\bigcup_{p \in I} B\left(x_{p} ; 10 r_{x_{p}}\right)$. Then

$$
\begin{gathered}
m\left(L_{n}\right) \leq \sum_{p \in I} \frac{\pi^{d / 2} \cdot 5^{d} r_{x_{p}}^{d}}{\Gamma(1+d / 2)} \leq 5^{d} \cdot \frac{2}{n} \sum_{p \in I} \int_{B\left(x_{p} ; r_{x_{p}}\right)} \Phi_{n}(y) d y \\
\leq 5^{d} \cdot \frac{2}{n} \sum_{p \in I} \int_{B\left(x_{p} ; r_{x_{p}}\right)} \Phi_{n}(y) d y \leq \frac{2 \cdot 5^{d}}{n} \int_{\Omega} \Phi_{n}(y) d y=\frac{2 \cdot 5^{d} \alpha_{n}}{n}, \\
m\left(L_{n}^{\prime}\right) \leq \sum_{p \in I} \frac{\pi^{d / 2} \cdot 10^{d} r_{x_{p}}^{d}}{\Gamma(1+d / 2)} \leq \frac{2 \cdot 10^{d} \alpha_{n}}{n} .
\end{gathered}
$$

Let $T_{n}=\Omega \backslash L_{n}^{\prime}$. First we prove that the inequality

$$
\int_{T_{n}}\left|R_{j, \Omega}\left(\Phi_{n}-\Phi_{n}^{*}\right)(x)\right| d x<C_{2} \cdot \alpha_{n}
$$

holds, where $C_{2}$ is a constant, independent of $n$. Denote

$$
h_{n}(x)=R_{j, \Omega}\left(\Phi_{n}-\Phi_{n}^{*}\right)(x) .
$$

For any $x \in T_{n}$, we have

$$
\left|h_{n}(x)\right|=C_{(d)}\left|\int_{\Omega} \frac{x_{j}-y_{j}}{|x-y|^{d+1}}\left[\Phi_{n}(y)-\Phi_{n}^{*}(y)\right] d y\right|
$$




$$
\begin{gathered}
=C_{(d)}\left|\sum_{p \in I} \int_{G_{p}} \frac{x_{j}-y_{j}}{|x-y|^{d+1}}\left[\Phi_{n}(y)-\Phi_{n}^{*}(y)\right] d y\right| \\
\leq C_{(d)} \sum_{p \in I}\left|\int_{G_{p}} \frac{x_{j}-y_{j}}{|x-y|^{d+1}} \Phi_{n}(y) d y-\int_{G_{p}} \frac{x_{j}-y_{j}}{|x-y|^{d+1}} \Phi_{n}^{*}(y) d y\right| .
\end{gathered}
$$

It follows from the integral mean value theorem that for any $p \in I$ there are points $y^{(p)}, \tilde{y}^{(p)} \in$ $B\left(x_{p} ; 5 r_{x_{p}}\right)$, such that

$$
\begin{aligned}
& \int_{G_{p}} \frac{x_{j}-y_{j}}{|x-y|^{d+1}} \Phi_{n}(y) d y=\frac{x_{j}-y_{j}^{(p)}}{\left|x-y^{(p)}\right|^{d+1}} \cdot \int_{G_{p}} \Phi_{n}(y) d y, \\
& \int_{G_{p}} \frac{x_{j}-y_{j}}{|x-y|^{d+1}} \Phi_{n}^{*}(y) d y=\frac{x_{j}-\tilde{y}_{j}^{(p)}}{\left|x-\tilde{y}^{(p)}\right|^{d+1}} \cdot \int_{G_{p}} \Phi_{n}^{*}(y) d y .
\end{aligned}
$$

Then from (3.6) and (3.9), we obtain that

$$
\left|h_{n}(x)\right| \leq C_{(d)} \sum_{p \in I}\left|\frac{x_{j}-y_{j}^{(p)}}{\left|x-y^{(p)}\right|^{d+1}}-\frac{x_{j}-\tilde{y}_{j}^{(p)}}{\left|x-\tilde{y}^{(p)}\right|^{d+1}}\right| \cdot \int_{G_{p}} \Phi_{n}(y) d y .
$$

Since for any $y, \tilde{y} \in B\left(x_{p} ; 5 r_{x_{p}}\right)$ and $x \in T_{n}$, the inequality

$$
\begin{gathered}
\left|\frac{x_{j}-y_{j}}{|x-y|^{d+1}}-\frac{x_{j}-\tilde{y}_{j}}{|x-\tilde{y}|^{d+1}}\right| \\
\leq \frac{\left|\left(x_{j}-y_{j}\right) \cdot\left[|x-\tilde{y}|^{d+1}-|x-y|^{d+1}\right]-\left(y_{j}-\tilde{y}_{j}\right) \cdot\right| x-\left.y\right|^{d+1} \mid}{|x-y|^{d+1} \cdot|x-\tilde{y}|^{d+1}} \\
\leq \frac{\left|x_{j}-y_{j}\right| \cdot|| x-\tilde{y}|-| x-y|| \cdot \sum_{k=0}^{d}|x-\tilde{y}|^{k} \cdot|x-y|^{d-k}}{|x-y|^{d+1} \cdot|x-\tilde{y}|^{d+1}}+\frac{\left|y_{j}-\tilde{y}_{j}\right|}{|x-\tilde{y}|^{d+1}} \\
\leq \frac{(d+1) \cdot 10 r_{x_{p}} \cdot 2^{d+1}}{\left|x-x_{p}\right|^{d+1}}+\frac{10 r_{x_{p}} \cdot 2^{d+1}}{\left|x-x_{p}\right|^{d+1}}=\frac{10(d+2) \cdot 2^{d+1} \cdot r_{x_{p}}}{\left|x-x_{p}\right|^{d+1}}
\end{gathered}
$$

holds, then it follows from (3.10) that

$$
\begin{gathered}
\left|h_{n}(x)\right| \leq C_{(d)} \sum_{p \in I} \frac{10(d+2) \cdot 2^{d+1} \cdot r_{x_{p}}}{\left|x-x_{p}\right|^{d+1}} \cdot \int_{G_{p}} \Phi_{n}(y) d y \\
\leq C_{(d)} \sum_{p \in I} \frac{10(d+2) \cdot 2^{d+1} \cdot r_{x_{p}}}{\left|x-x_{p}\right|^{d+1}} \cdot\left(\frac{1}{2} \cdot \frac{\pi^{d / 2}}{\Gamma(1+d / 2)} r_{x_{p}}^{d} \cdot n\right) \\
=C_{3} \cdot n \cdot \sum_{p \in I} \frac{r_{x_{p}}^{d+1}}{\left|x-x_{p}\right|^{d+1}},
\end{gathered}
$$

where $C_{3}=\frac{\Gamma((d+1) / 2) \cdot 10(d+2) \cdot 2^{d}}{\pi^{1 / 2} \cdot \Gamma(1+d / 2)}$. From this, we get that

$$
\begin{aligned}
& \int_{T_{n}}\left|h_{n}(x)\right| d x \leq C_{3} \cdot n \cdot \sum_{p \in I} r_{x_{p}}^{d+1} \int_{T_{n}} \frac{d x}{\left|x-x_{p}\right|^{d+1}} \\
\leq & C_{3} \cdot n \cdot \sum_{p \in I} r_{x_{p}}^{d+1} \int_{\left\{x \in R^{d}:\left|x-x_{p}\right| \geq 10 r_{x_{p}}\right\}} \frac{d x}{\left|x-x_{p}\right|^{d+1}}
\end{aligned}
$$




$$
\begin{gathered}
=C_{3} \cdot n \cdot \sum_{p \in I} r_{x_{p}}^{d+1} \cdot \frac{2 \pi^{d / 2}}{\Gamma(d / 2)} \int_{10 r_{x_{p}}}^{+\infty} \frac{d r}{r^{2}}=\frac{C_{3} \pi^{d / 2}}{5 \Gamma(d / 2)} \cdot n \cdot \sum_{p \in I} r_{x_{p}}^{d} \\
=\frac{C_{3} \pi^{d / 2}}{5 \Gamma(d / 2)} \cdot n \cdot \frac{2 \alpha_{n}}{n} \cdot \frac{\Gamma(1+d / 2)}{\pi^{d / 2}}=\frac{C_{3} d}{5} \alpha_{n} .
\end{gathered}
$$

That is, inequality (3.8) is satisfied.

We represent the function $f(x)$ in the form

$$
f(x)=[f(x)]^{n}+\Phi_{n}^{*}(x)+\left[\Phi_{n}-\Phi_{n}^{*}\right](x) .
$$

Let us prove that the equality

$$
\lim _{n \rightarrow \infty} \int_{T_{n}} g(x)\left(R_{j, \Omega} f\right)(x) d x=-\int_{\Omega} f(x)\left(R_{j, \Omega} g\right)(x) d x .
$$

holds. Consider the integral

$$
\begin{gathered}
\int_{T_{n}} g(x)\left(R_{j, \Omega} f\right)(x) d x \\
=\int_{T_{n}} g(x)\left\{\left(R_{j, \Omega}[f]^{n}\right)(x)+\left(R_{j, \Omega} \Phi_{n}^{*}\right)(x)+R_{j, \Omega}\left(\Phi_{n}-\Phi_{n}^{*}\right)(x)\right\} d x \\
=\int_{T_{n}} g(x)\left(R_{j, \Omega}[f]^{n}\right)(x) d x+\int_{T_{n}} g(x)\left(R_{j, \Omega} \Phi_{n}^{*}\right)(x) d x \\
+\int_{T_{n}} g(x) R_{j, \Omega}\left(\Phi_{n}-\Phi_{n}^{*}\right)(x) d x=S_{1}+S_{2}+S_{3} .
\end{gathered}
$$

By (3.4) for square integrable functions, we obtain

$$
\begin{gathered}
S_{1}=\int_{T_{n}} g(x)\left(R_{j, \Omega}[f]^{n}\right)(x) d x \\
=\int_{\Omega} g(x)\left(R_{j, \Omega}[f]^{n}\right)(x) d x-\int_{L_{n}^{\prime}} g(x)\left(R_{j, \Omega}[f]^{n}\right)(x) d x \\
=-\int_{\Omega}[f(x)]^{n}\left(R_{j, \Omega} g\right)(x) d x-\int_{L_{n}^{\prime}} g(x)\left(R_{j, \Omega}[f]^{n}\right)(x) d x=S_{1}^{(1)}+S_{1}^{(2)} .
\end{gathered}
$$

Since$$
\left|S_{1}^{(2)}\right|=\left|\int_{L_{n}^{\prime}} g(x)\left(R_{j, \Omega}[f]^{n}\right)(x) d x\right| \leq \int_{L_{n}^{\prime}}\left|\left(R_{j, \Omega}[f]^{n}\right)(x)\right| d x
$$$$
\leq\left[m\left(L_{n}^{\prime}\right) \cdot \int_{\Omega}\left(R_{j, \Omega}[f]^{n}\right)^{2}(x) d x\right]^{1 / 2}
$$$$
\leq C^{(2)}\left[m\left(L_{n}^{\prime}\right) \cdot \int_{\Omega}\left([f(x)]^{n}\right)^{2} d x\right]^{1 / 2}
$$$$
\leq C^{(2)}\left[n \cdot m\left(L_{n}^{\prime}\right) \cdot \int_{\Omega} f(x) d x\right]^{1 / 2},
$$

then it follows from (3.7) that

$$
\begin{aligned}
\lim _{n \rightarrow \infty} S_{1} & =-\lim _{n \rightarrow \infty} \int_{\Omega}[f(x)]^{n}\left(R_{j, \Omega} g\right)(x) d x \\
& =-\int_{\Omega} f(x)\left(R_{j, \Omega} g\right)(x) d x .
\end{aligned}
$$


For the integral $S_{2}$, we also have

$$
\begin{gathered}
S_{2}=\int_{T_{n}} g(x)\left(R_{j, \Omega} \Phi_{n}^{*}\right)(x) d x \\
=\int_{\Omega} g(x)\left(R_{j, \Omega} \Phi_{n}^{*}\right)(x) d x-\int_{L_{n}^{\prime}} g(x)\left(R_{j, \Omega} \Phi_{n}^{*}\right)(x) d x \\
=-\int_{\Omega} \Phi_{n}^{*}(x)\left(R_{j, \Omega} g\right)(x) d x-\int_{L_{n}^{\prime}} g(x)\left(R_{j, \Omega} \Phi_{n}^{*}\right)(x) d x=S_{2}^{(1)}+S_{2}^{(2)} .
\end{gathered}
$$

The following estimations are valid.

$$
\begin{gathered}
\left|S_{2}^{(1)}\right|=\left|\int_{\Omega} \Phi_{n}^{*}(x)\left(R_{j, \Omega} g\right)(x) d x\right| \leq \int_{\Omega}\left|\Phi_{n}^{*}(x)\left(R_{j, \Omega} g\right)(x)\right| d x \\
\leq \int_{\Omega} \Phi_{n}^{*}(x) d x=\int_{\Omega} \Phi_{n}(x) d x=\alpha_{n} \\
\left|S_{2}^{(2)}\right|=\left|\int_{L_{n}^{\prime}} g(x)\left(R_{j, \Omega} \Phi_{n}^{*}\right)(x) d x\right| \leq \int_{L_{n}^{\prime}}\left|\left(R_{j, \Omega} \Phi_{n}^{*}\right)(x)\right| d x \\
\leq\left[m\left(L_{n}^{\prime}\right) \cdot \int_{\Omega}\left(R_{j, \Omega} \Phi_{n}^{*}\right)^{2}(x) d x\right]^{1 / 2} \leq C^{(2)}\left[m\left(L_{n}^{\prime}\right) \cdot \int_{\Omega}\left(\Phi_{n}^{*}(x)\right)^{2} d x\right]^{1 / 2} \\
\leq C^{(2)}\left[\frac{5^{d} n}{2} \cdot m\left(L_{n}^{\prime}\right) \cdot \int_{\Omega} \Phi_{n}^{*}(x) d x\right]^{1 / 2}=C^{(2)}\left[\frac{5^{d} n}{2} \cdot m\left(L_{n}^{\prime}\right) \cdot \alpha_{n}\right]^{1 / 2} .
\end{gathered}
$$

Then it follows from (3.7) that

$$
\lim _{n \rightarrow \infty} S_{2}=0 \text {. }
$$

To estimate the integral $S_{3}$, we need to apply the inequality (3.8):

$$
\begin{aligned}
& \left|S_{3}\right|=\left|\int_{T_{n}} g(x) R_{j, \Omega}\left(\Phi_{n}-\Phi_{n}^{*}\right)(x) d x\right| \\
& \quad \leq \int_{T_{n}}\left|g(x) R_{j, \Omega}\left(\Phi_{n}-\Phi_{n}^{*}\right)(x)\right| d x \\
& \leq \int_{T_{n}}\left|R_{j, \Omega}\left(\Phi_{n}-\Phi_{n}^{*}\right)(x)\right| d x<C_{2} \cdot \alpha_{n} .
\end{aligned}
$$

This implies the equality

$$
\lim _{n \rightarrow \infty} S_{3}=0 \text {. }
$$

From the equalities (3.13), (3.14), (3.15) and (3.16), we obtain the equality (3.12). It remains to prove the equality

$$
\text { (A) } \int_{\Omega} g(x)\left(R_{j, \Omega} f\right)(x) d x=\lim _{n \rightarrow \infty} \int_{T_{n}} g(x)\left(R_{j, \Omega} f\right)(x) d x .
$$

Consider the difference of integrals

$$
\begin{gathered}
\int_{T_{n}} g(x)\left(R_{j, \Omega} f\right)(x) d x-\int_{\Omega}\left[g(x)\left(R_{j, \Omega} f\right)(x)\right]^{n} d x \\
=-\int_{L_{n}^{\prime}}\left[g(x)\left(R_{j, \Omega} f\right)(x)\right]^{n} d x+
\end{gathered}
$$




$$
+\int_{T_{n}}\left\{g(x)\left(R_{j, \Omega} f\right)(x)-\left[g(x)\left(R_{j, \Omega} f\right)(x)\right]^{n}\right\} d x=S^{(1)}+S^{(2)} .
$$

From the inequality $\left|S^{(1)}\right| \leq n \cdot m\left(L_{n}^{\prime}\right)$, it follows that

$$
\lim _{n \rightarrow \infty} S^{(1)}=0 .
$$

Denote $\sigma_{n}=\left\{x \in \Omega:\left|g(x)\left(R_{j, \Omega} f\right)(x)\right|>n\right\}$. Since

$$
m\left\{x \in \Omega:\left|\left(R_{j, \Omega} f\right)(x)\right|>n\right\}=o\left(\frac{1}{n}\right), n \rightarrow \infty,
$$

then $m\left(\sigma_{n}\right)=o\left(\frac{1}{n}\right), n \rightarrow \infty$. Using (3.8) and (3.11), we obtain

$$
\begin{gathered}
\left|S^{(2)}\right| \leq \int_{T_{n} \cap \sigma_{n}}\left|g(x)\left(R_{j, \Omega} f\right)(x)\right| d x \\
\leq \int_{T_{n} \cap \sigma_{n}}\left|\left(R_{j, \Omega} f\right)(x)\right| d x \leq \int_{\sigma_{n}}\left|\left(R_{j, \Omega}[f]^{n}\right)(x)\right| d x \\
+\int_{\sigma_{n}}\left|\left(R_{j, \Omega} \Phi_{n}^{*}\right)(x)\right| d x+\int_{T_{n}}\left|R_{j, \Omega}\left(\Phi_{n}-\Phi_{n}^{*}\right)(x)\right| d x \\
\leq\left[m\left(\sigma_{n}\right) \cdot \int_{\Omega}\left(R_{j, \Omega}[f]^{n}\right)^{2}(x) d x\right]^{1 / 2} \\
+\left[m\left(\sigma_{n}\right) \cdot \int_{\Omega}\left(R_{j, \Omega} \Phi_{n}^{*}\right)^{2}(x) d x\right]^{1 / 2}+C \cdot \alpha_{n} \\
\leq c_{2}\left[m\left(\sigma_{n}\right) \cdot \int_{\Omega}\left([f(x)]^{n}\right)^{2} d x\right]^{1 / 2} \\
+c_{2}\left[m\left(\sigma_{n}\right) \cdot \int_{\Omega}\left(\Phi_{n}^{*}(x)\right)^{2} d x\right]^{1 / 2}+C \cdot \alpha_{n} \\
\leq c_{2}\left[n \cdot m\left(\sigma_{n}\right) \cdot \int_{\Omega} f(x) d x\right]^{1 / 2} \\
+c_{2}\left[\frac{5^{d} n}{2} \cdot m\left(\sigma_{n}\right) \cdot \int_{\Omega} \Phi_{n}^{*}(x) d x\right]^{1 / 2}+C \cdot \alpha_{n} .
\end{gathered}
$$

It follows that

$$
\lim _{n \rightarrow \infty} S^{(2)}=0 .
$$

From the equalities (3.18), (3.19) and (3.20), we obtain the equality (3.17). Theorem 3.1 is proved.

\section{Acknowledgments}

The authors are very grateful to the referees for many useful comments and suggestions that improved the original manuscript. 


\section{REFERENCES}

[1] A. B. Aleksandrov: A-integrability of the boundary values of harmonic functions. Math. Notes 30(1) (1981), 515-523.

[2] R. A. Aliev: $N^{ \pm}$-integrals and boundary values of Cauchy-type integrals of finite measures. Sbornik: Mathematics 205(7) (2014), 913-935.

[3] R. A. Aliev: On properties of Hilbert transform of finite complex measures. Complex Analysis and Operator Theory 10(1) (2016), 171-185.

[4] R. A. Aliev: Riesz's equality for the Hilbert transform of the finite complex measures. Azerb. J. Math. 6(1) (2016), 126-135.

[5] R. A. Aliev: Representability of Cauchy-type integrals of finite complex measures on the real axis in terms of their boundary values. Complex Variables and Elliptic Equations 62(4) (2017), 536-553.

[6] R. A. Aliev, K. I. Nebiyeva: The A-integral and Restricted Complex Riesz Transform. Azerbaijan Journal of Mathematics 10(1) (2020), 209-221.

[7] A. S. Besicovitch: On a general metric property of summable functions. J. London Math. Soc. 1(2) (1926), 120-128.

[8] M. P. Efimova: On the properties of the Q-integral. Math. Notes 90(3-4) (2011), 322-332.

[9] M. P. Efimova: The sufficient condition for integrability of a generalized Q-integral and points of integrability. Moscow Univ. Math. Bull. 70(4) (2015), 181-184.

[10] L. C. Evans, R. F. Gariepy: Measure theory and fine properties of functions. CRC Press, Boca Raton (1992).

[11] M. A. Ragusa: Elliptic boundary value problem in Vanishing Mean Oscillation hypothesis. Comment. Math. Univ. Carolin 40(4) (1999), 651-663.

[12] M. A. Ragusa: Necessary and sufficient condition for a VMO function. Applied Mathematics and Computation 218(24) (2012), 11952-11958.

[13] T. S. Salimov: The A-integral and boundary values of analytic functions. Math. USSR-Sbornik 64(1) (1989), $23-40$.

[14] V. A. Skvortsov: A-integrable martingale sequences and Walsh series. Izvestia: Math. 65(3) (2001), 607-616.

[15] E. M. Stein: Singular Integrals and Differentiability Properties of Functions. Princeton University Press, Princeton (1970).

[16] E. C. Titchmarsh: On conjugate functions. Proc. London Math. Soc. 9 (1929), 49-80.

[17] P. L. Ul'yanov: The A-integral and conjugate functions. Mathematics, vol.7 (1956), Uch. Zap. Mosk. Gos. Univ. 181, 139-157, (in Russian).

[18] P. L. Ul'yanov: Integrals of Cauchy type. Twelve Papers on Approximations and Integrals. Amer. Math. Soc. Trans. 2(44) (1965), 129-150.

\section{BAKU STATE UNIVERSITY,}

BAKU, AZERBAIJAN,

INSTITUTE OF MATHEMATICS AND MECHANICS,

NAS OF AZERBAIJAN, BAKU, AZERBAIJAN

ORCID: 0000-0003-1709-902X

E-mail address: aliyevrashidemail.ru

KHAZAR UNIVERSITY,

BAKU, AZERBAIJAN

ORCID: 0000-0001-8412-7305

E-mail address: xanim.nebiyeva@gmail.com 